\title{
Low Power Circuit Design for Footed Quasi Resistance Scheme In 45NM VLSI Technology
}

\author{
Telugu Satyanarayana ${ }^{a}$, Dr. Mukesh Tiwari ${ }^{b}$ and Dr. Vundela Padmanabha Reddy \\ a Research Scholar, Dept. of Electronics and Communication Engineering, \\ Sri Satya Sai University of Technology \& Medical Sciences, Sehore, Bhopal Indore Road, Madhya Pradesh, India \\ ${ }^{\mathbf{b}}$ Research Guide, Dept. of Electronics and Communication Engineering, \\ Sri Satya Sai University of Technology \& Medical Sciences, Sehore, Bhopal Indore Road, Madhya Pradesh, India \\ ${ }^{c}$ Research Co- Guide,Department of ECE, Institute of Aeronautical Engineering, Hyderabad
}

Article History: Received: 11 January 2021; Accepted: 27 February 2021; Published online: 5 April 2021

\begin{abstract}
Low power has arisen as a chief topic in these days and hardware enterprises. Power dissipation has become a significant thought as execution and zone of VLSI Chip plan. In this paper, a design of low power for footed quasi resistance scheme in 45nanometer VLSI technology, using appropriate standard digital gates with 45nm technology, considering footed quasi resistance technique for nanoscales is introduced. Transition of logic 1 and 0 is the main problem in the cascading circuits, this problem can solved by employing a basic inverter called as Domino logic at output.Due to the precharge propagation the power dissipation is observed in domino logic, this will be resolved using PDB (Pseudo Dynamic Buffer) model. With the help of PDB nearly $67 \%$ of power saved. Even though PDB is succeeded in precharge propagation, it fails in logic transition, this may results erroneous output during cascading. With contracting technology, power utilization can decreased and over all power of the executives on chip are the critical difficulties below 100nm because of expanded intricacy. In this paper execution of low power circuit scheme for footed quasi resistance plot in 45nm VLSI technology. In this paper we will actualize and recreate low power circuit scheme for footed quasi resistance plot in 45nm VLSI technology.
\end{abstract}

KEY WORDS: 45 nanometer technology, PDB (Psuedo dynamic buffer),GaN on Si pin diodes, Footed Quasi Resistance.

\section{Introduction}

Low power and high speed logic circuits areattaining more attention than any device taking in consideration. Since for delay and optimized power, several logic families are designed [1]. The main aim of these logic circuits is to reduce delay, speed of operation and to decrease the power dissipation. In present scenario, the major role played by Dynamiclogic circuits, theseare used in the circuit to decrease the power dissipation.

In comparison with static CMOS, the area occupied by Dynamic circuit is less [2].These logic circuits can designed with nearly half of transistors used in designing of static CMOS circuits. Total Power dissipated in a CMOS circuit is amount of dynamic power, impede and static or spillage power. The methods which are used to decrease power scattering are not the restriction to dynamic power. This section discuss about circuit and logic configuration ways to deal with limit dynamic, spillage and short out power dissemination.In order to prevent the erroneous output during the design of cascade dynamic circuits, the logic circuits of Domino are modified as dynamic logic at output along with extended simple inverter of static CMOS.To control operations of domino logic, a clock signal is used same as in dynamic circuits.

The operations of a Domino logic circuit are dividesinto mainly two phases. The first phase is called as precharge phase, if clock is at low, $\mathrm{V}_{\mathrm{DD}}$ at the output of the parasitic capacitance charges it, that was present before a simple static inverter. This results makes output domino logic circuit as 0 logic and in evolution stage, that is at second stage if the clock high,the capacitance of load will maintain or discharge as per the input. In precharge phase a domino logic circuit uses extra inverter to prevent the logic 0 , and logic 1 transitions in evaluation phase, this means at capacitance of output inverter in first phase i.e, pre-charge phase, charging as well as discharging can takes place, if its converse evolution phase has logic 0 as output.This may be cause a significant wastage of power.This problem solved by many methods, in which efficient method is PDB, it saves upto $67 \%$ of power.

The exacting impediment on power dissemination in convenient hardware applications, for example, PDAs and tablet PCs must be met by the VLSI chip originator while as yet meeting the computational necessities. While remote gadgets are quickly advancing toward the shopper hardware market, a key plan imperative for compact activity specifically the total power utilization of the gadget must be tended to. Diminishing the total power utilization in such frameworks is significant since it is alluring to augment the run time with least necessities on size, battery life and weight distributed to batteries.

So the main factor to consider while planning SoC for convenient gadgets is 'low power scheme' [4].The developing business sector of portables, for example, phones, gaming consoles and battery-powered electronic frameworks requests microelectronic circuit's plan with super low power scattering, therefore VLSI technology came into scenario. As the incorporation, size, and unpredictability of the chips keep on expanding, the trouble in giving satisfactory cooling may either add critical expense or cutoff the usefulness of the figuring frameworks which utilize those integrated circuits. Thus, the VLSI technology is used in proposed system based on $45 \mathrm{~nm}$ technology to overcome power dissipation [5]. 


\section{PSUEDO DYNAMIC BUFFER}

a pseudo dynamic buffer method is able to eliminate the pre-charge pulse propagation. Here, in this section different types of domino logics are explained, it's because domino logic circuits plays major role in power dissipation in integrated circuits. This circuit helps in reduction of precharge propagation.

\subsection{Conventional Domino Logic Circuit}

Designing of a conventional domino logic can be done by using two $\mathrm{N}$ type (pull down) and $\mathrm{P}$ type (pull up) networks. A simple dynamic logic circuit is as same as a conventional domino logic which designed with a simple buffer of $\mathrm{N}$ type network, which operates in two phases, as discussed earlier they are precharge phase and evaluation phase. Clock signal distinguishes these two phases.If clock signal is at low, the PMOS transistor at output charges the capacitance node to $\mathrm{V}_{\mathrm{DD}}$ is known as precharge phase. If clock signal is at high (one), the NMOS transistor at output is high and node $\mathrm{x}$ voltage depends on $\mathrm{N}$ type networks input, and this is known as evaluation mode [6].Moreover, if signal (input) is high (1), thus the node voltages are given to both precharge and evaluation phases. The voltage at $\mathrm{V}_{\mathrm{DD}}$ is charged during evolution phase and discharged during pre charge phase which leads to pre charge propagation, which results in higher power consumption at output buffer. This precharge pulse propagation issolved by using different techniques like truesingle phase c1k model and pseudo dynamic buffer.

\subsection{Psuedo Dynamic Buffer Domino Logic}

In PDB model source of transistor Qs in outputinverter is connected to node $\mathrm{Y}$ instead of ground asshown in figure (1). For this particular model when input A islogic 1, evaluation and precharge will lead to followingcase. During evaluation phase, node $\mathrm{X}$ will discharge tologic 0 which makes PMOS transistor Q4 ON and

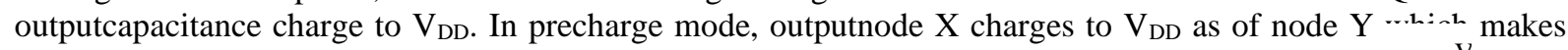
NMOStransistor Qs OFF.Transistor Qs operates as following. In prechargemode previously sc V: Qs is connected to ground,logic high at node $X$ turns $O N$ transistor Qs because $V_{g}-V_{s}\left(V_{g s}\right)$ is greater than threshold voltage $\left(\mathrm{V}_{\mathrm{th}}\right)$ in PDB sourceof transistor Qs is connected to node $\mathrm{Y}$ which makes Qs operation depends on node $\mathrm{X}$ only in precharge mode by virtue of when c1k signal is low transistor Q3 turns ONmakes source of transistor Qs connected to ground as indomino logic circuits.

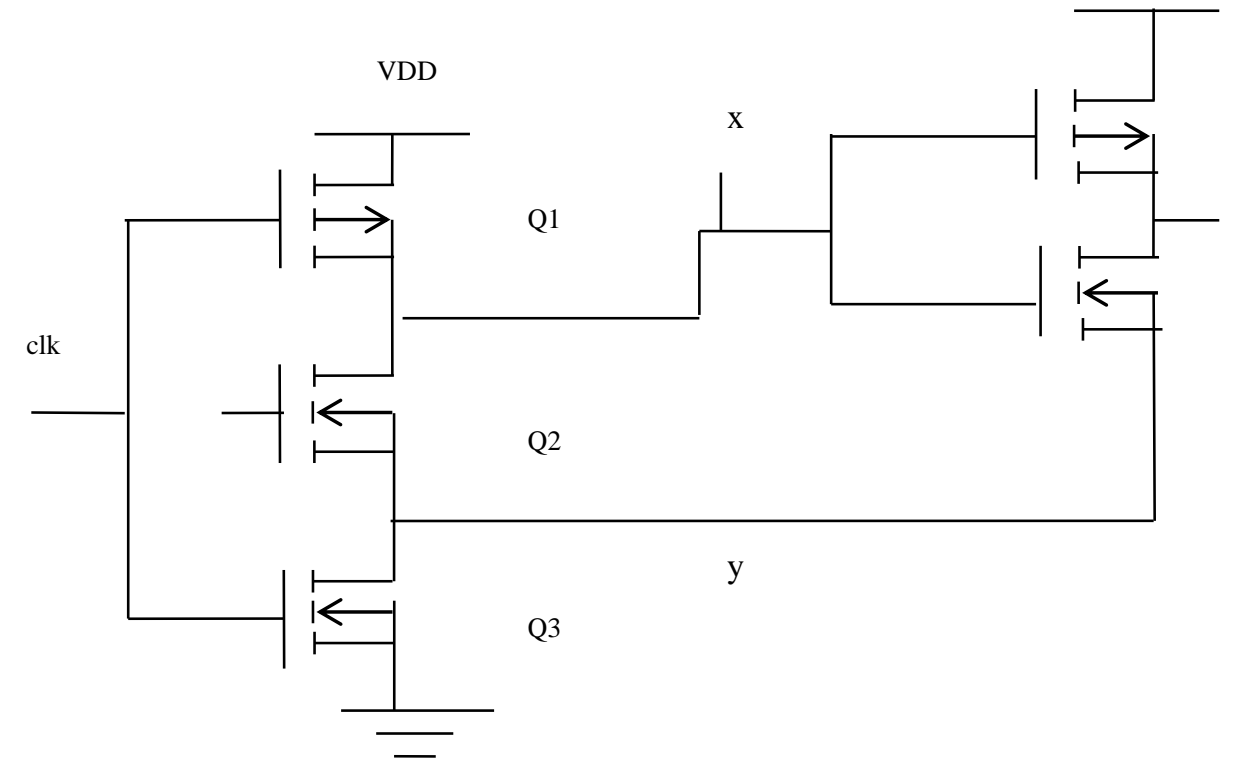

Q4

Q5

Fig. 1:Psuedo Dynamic Buffer Domino Logic

But when c1k signal is high,transistor Qs operation depends on both voltage at node Xand input logic applied for domino logic circuit. But in PDB model there is small problem when logic0 is applied as input after logic 1 in evaluation state. This is because output parasiticcapacitance charge accumulated in evaluation state wheninput $\mathrm{A}$ is logic 1 doesn't have discharge path in prechargestate when input $\mathrm{A}$ is logic 0 . Because of this, logic 1 tologic 0 transition which is not a suitable input for dynamiclogic circuits.

\subsection{Fault Output in Cascaded Circuits}

After overcoming precharge pulse propagation PDBcircuit catch on major problem: straightforward perusal ofpseudo dynamic buffer logic to design more complexboolean functions gets faulty output logic [7-8]. The issue isbest exemplified with the two cascaded pull downn-type pseudo dynamic buffer circuits in reducing of power consumption. Figure (2) shows the circuit of fault output in cascaded circuits this eliminates cascading problem.During the precharge phase (i.e., precharge phase afterlogic high in evaluation phase), the outputs of 
both buffersare already charged to $\mathrm{V}_{\mathrm{DD}}$. Consider that the primary inputsignal $\mathrm{A}$ makes a logic 1 to logic 0 transition. On therising verge of the clk signal, output 01 starts to discharge.

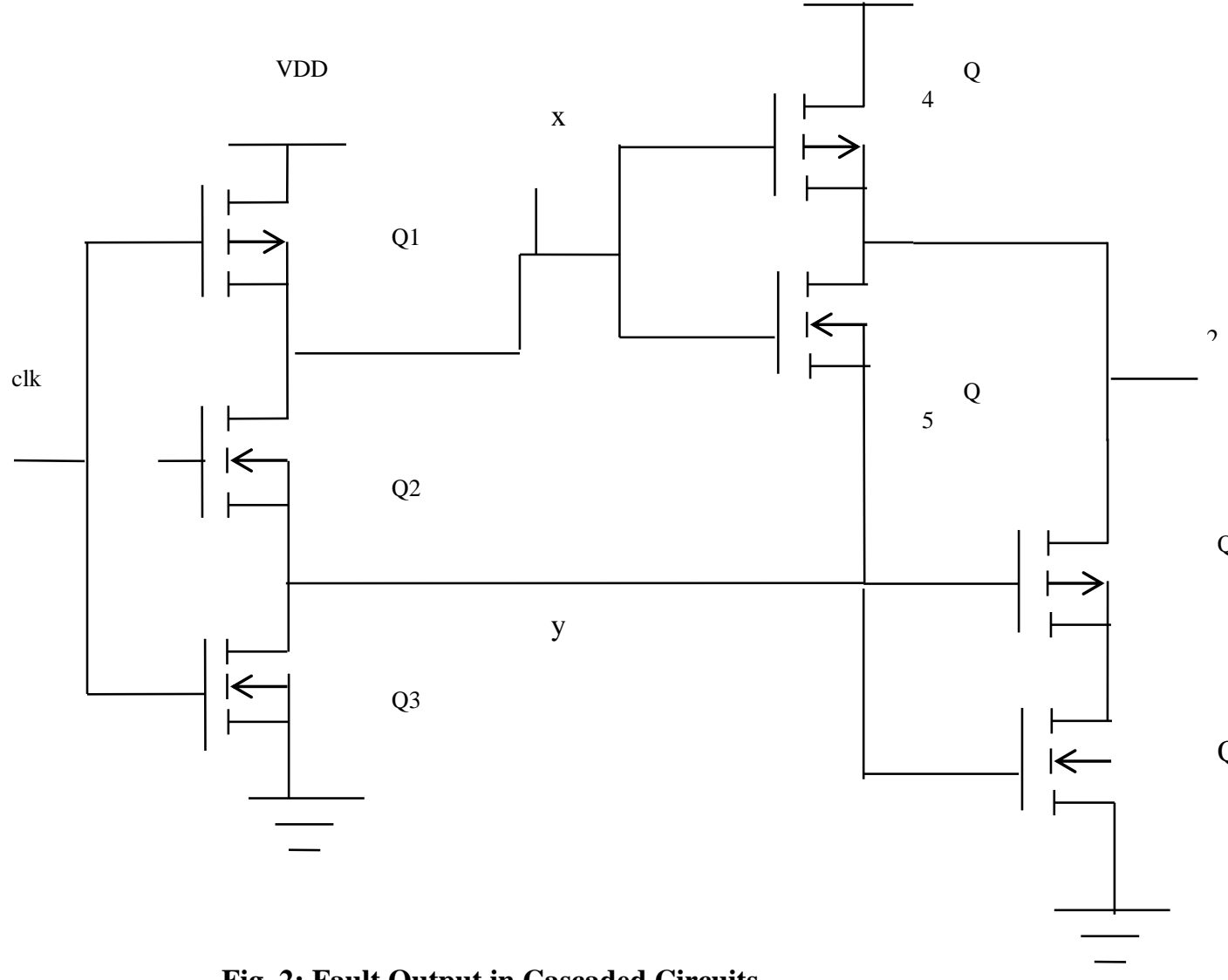

$\mathrm{O}$

The final output $\mathrm{O}$ should be logic low as its expectedinput is logic low. Despite, there is a small propagationdelay for the input to discharge 01 to logic low. Therefore,the precharged voltage at node $\mathrm{Z}$ gets discharged. By 01cross the threshold of the transistor Q7 node voltage at Zgets discharged through Q7 and Q6. This makes finaloutput as logic high, correct logic level can't be retrievebecause PDB relies on parasitic capacitance voltage. Thislead to erroneous output logic in cascaded PDB logiccircuits. Therefore this problem of capacitance and high output is reduced by employing GaN on Si pin diodes of vertical quasi and FQR technique. The proposed system can recreate the low power plan for quasi foot resistance in $45 \mathrm{~nm}$ technology [9-10].

\section{FOOTEDQUASIRESISTANCESCHEME}

To implement low power circuit for footed quasi resistance the main scheme is used is FQR explained in this section along with layout of proposed system.

\subsection{Footed Quasi Resistance (FQR) model}

The foregoing section illustrates the issue of cascading problem due to logic 1 to logic 0 transition at output node. Suggested footed quasi resistance model overcomes this cascading issue using structure. In proposed circuit used depletion PMOS and NMOS, which are driven by same input node Y. when logic 1 is at node $\mathrm{Y}$ depletion PMOS is OFF which makes quasi resistance acting as open circuit, same in case of logic 0 at node $\mathrm{Y}$ but this time depletion NMOS is OFF which resuIts same. When input A is logic 1 in precharge phase node $\mathrm{Y}$ is connected to $\mathrm{V}_{\mathrm{DD}}$ and in evaluation phaseit is connected to ground which makes footed quasiresistance works as PDB only. When input A is logic 0 the footed quasi resistance functionality in evaluation and precharge phase is:During precharge phase transistor Q2 and Q3 are OFF which will lead node Y as open circuit. Because we use depletion NMOS and PMOS in FQR which provides path to discharge output parasitic capacitance. During evaluation phase NMOS transistor Q3 is ON and NMOS transistor Q5 ON which makes output node logicO.In output voItage waveform is given where logic 1 to logic 0 transition is removed and is explained in figure(2). Here the proposed layout of low power quasi resistance is given in figure (3).

\subsection{GaN- on -Si pin diodes}

In comparison with the pin diodes which are grown $\mathrm{GaN}$, diodes which are grown on large area on Si substrate are considered as a enblers those can minimize the cost of using large scale inexpensive Si. These have major advantage over the Si CMOS manufacturing compatibility.In order to date both vertical diodes of quasi which are grown on Si substrate and fully vertical diodes those are grown on $\mathrm{GaN}$ are transferred to $\mathrm{Si}(100)$ thin films. 
After Mesa etching followed by metallization process, the diodes of vertical quasi both anode and cathode are formed side by side on epilayer's same side. The degradation of performance of devices are due to non uniform distribution of crowding effect of currents and electrical field under power operation. Due to this, long term reliability also decreases. In order to overcome this problem, fully vertical PIN diodes are fabricated on Si through bonding of metal.

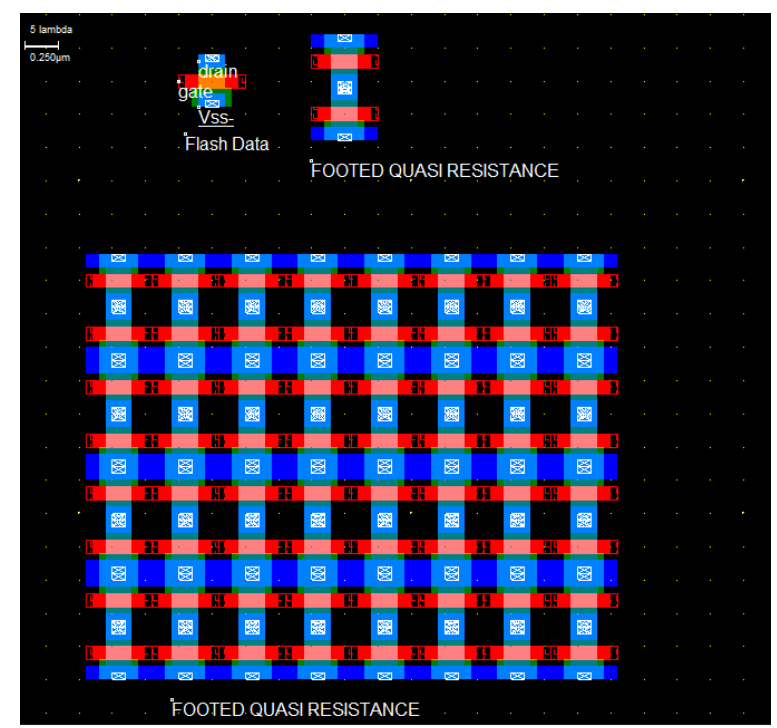

Fig. 3: Proposed layout of quasi resistance

There was far gap between theoretical and practical limits in the characteristics of devices. So there is a lot to improve the fully vertical PIN diodes because of its most complicated process. Anefficient technology thus required and comprehensive knowledge about both quasi vertical and fully vertical pin diodes configurations are highly desired in the process of developing of a low power circuit for quasi resistance. Power utilization in the versatile frameworks is also low. Power utilization is one of the significant components of VLSI circuit plan for CMOS, is the essential technology. The power utilization has become a key issue in VLSI circuit plan. The architects are needed to pick suitable strategies that fulfill the application and item needs. Lessening power dissemination changes from application to application.

This proposed system is designed by using $45 \mathrm{~nm}$ parameters of processtechnology; at low powers this may offers in turn high speed performance. The main innovation that related to the VLSI 45nm technology is like high-k gate oxide, and very low-k interconnectdielectric and metal-gate are described. Trends, chip fabrication,process design VLSI Technology includes, circuit design and real circuitparameters,electrical characteristics, building blocks of configuration, switching circuitry, CAD, silicon translation CAD, layout design practical experience are included in the process of VLSI technology.

The integration process, gates and diodes used in 45nm technology will help the in reduction of total heat dissipation. The power dispersal of a chip depends on its technology as well as on its execution for example on size, circuit style, working frequency, etc. Due to this technology patterns, transistor spillage power has expanded dramatically supply voltage scaling builds sub-limit spillage current, builds spillage power and represent various spillage in the VLSI plan. Hence static power has become a huge segment of the total power utilization. There are few VLSI procedures to lessen spillage power. Various procedures give a productive method to diminish spillage power, however hindrances limit the utilization of every strategy.

\section{RESULTS}

Low power circuit for footed quasi resistance is proposed and designed. The first step in fabrication of circuit in $45 \mathrm{~nm}$ VLSI technology is isolation of Sio2. It is shown in figure (4).

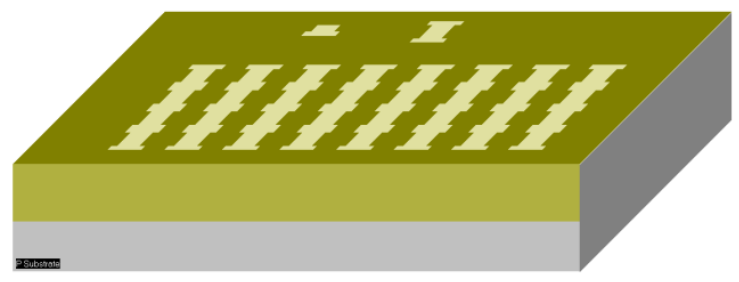

Fig. 4:Sio 2 isolation layout 
Second step is fabricating polysilicon on isolation layout is shown in figure (5).

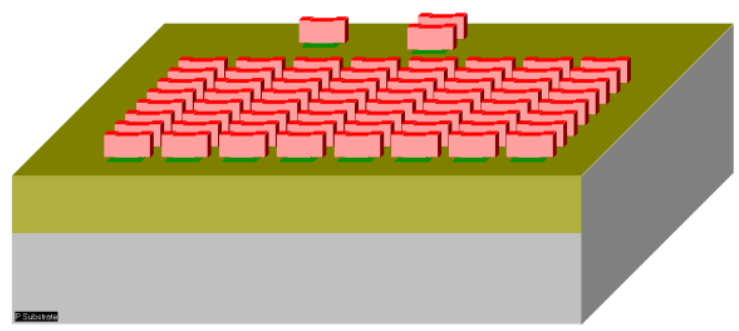

Fig. 5:Polysilicon layout

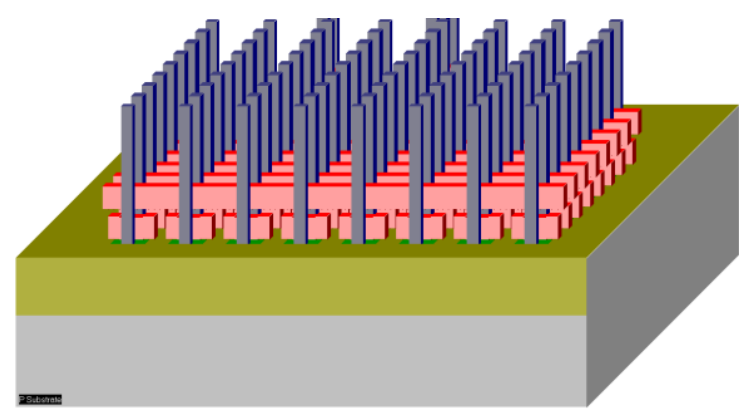

Fig. 6:Final layout

Final layout of the proposed system is shown in figure (6).

\section{CONCLUSION}

Along with elimination of precharge propagation problem, PDB encounters a new problem of cascading logic. In this proposed footed quasi resistance scheme which solves problem of cascading.57\% of power saved in FQR technique.The digital gates and $\mathrm{GaN}$ on $\mathrm{Si}$ Pin diode helps in reducing the resistance due to this less heat dissipation was occurred that leads to maximum throughput which in turn produces highly optimized circuits for footed quasi resistance in $45 \mathrm{~nm}$ VLSI design.Butthis process can compromise the power saving. This is worthwhile than PBD when considering our proposed system. By reducing power dissipation in circuit, makes a low power circuit for footed resistance. The proposed design of low power circuit for quasi resistance is designed.

\section{REFERENCES}

1. Eblabla, X. Li, D. J. Wallis, I. Guiney and K. Elgaid, "High-performance MMIC inductors for GaNon-low-resistivity silicon for microwave applications", IEEE Microw. Wireless Compon. Lett., vol. 28, no. 2, pp. 99-101, Feb. 2018.

2. Lu, G. Liu and K. Bai, "Critical transient processes of enhancement-mode GaN HEMTs in highefficiency and high-reliability applications", CES Transactions on Electrical Machines and Systems, vol. 1, no. 3, pp. 283-291, September 2017.

3. Chowdhury and U. K. Mishra, "Lateral and vertical transistors using the AlGaN/GaN heterostructure", IEEE Trans. Electron Devices, vol. 60, no. 10, pp. 3060-3066, Oct. 2013

4. Arora and J. D. Cressler, "Operating voltage constraints in 45-nm SOI nMOSFETs and cascode cores", IEEE Trans. Nucl. Sci., vol. 60, no. 1, pp. 132-139, Feb. 2013.

5. Richard, A. Siligaris, F. Badets, C. Dehos, C. Dufis, P. Busson, P. Vincent, D. Belot, and P. Urard, "A 17.5 GHz-to-20.94 GHz and 35-to-41.88 GHz PLL in $65 \mathrm{~nm}$ CMOS for wireless HD application," in IEEE Int. Solid-State Circuits conf. Dig., Feb. 2010, pp. 252-253., IEEE Trans. Nucl. Sci., vol. 60, no. 1, pp. 132-139, Feb. 2013

6. V. Kshirsagar and M. V. Vyawahare, "FPGA Implementation of High speed VLSI Architectures for AES Algorithm", 2012 Fifth International Conference on Emerging Trends in Engineering and Technology, pp. 239-242, Nov. 2012.

7. J. C. Chen et al., IEEE Transactions on CPMT, vol. 2, no. 6, pp. 956-963, June 2012.

8. Martinez, M. Aldegunde, N. Seoane, A. Brown, J. Barker and A. Asenov, "Quantum-transport study on the impact of channel length and cross sections on variability induced by random discrete dopants in narrow gate-all-around silicon nanowire transistors", IEEE Trans. Electron Dev, vol. 58, no. 8, pp. 2209-2217, Aug 2011. 
9. Taiki Uemura, Ryo Tanabe, Yoshiharu Tosaka, Shigeo Satoh, "Using Low Pass Filters in Mitigation Techniques against Single-Event Transients in 45nm Technology LSIs", On-Line Testing Symposium 2008. IOLTS '08. 14th IEEE International, pp. 117-122, 2008.

10. P. Giblin and Jonathan M. Williams, "Automation of a Coaxial Bridge for Calibration of AC Resistors" IEEE Trans. Instrum. Meas., vol. 56, no. 2, pp. 373-377, April 2007.

11. E. Gusev, V. Narayanan and M. Frank, IBM Res. \& Dev., vol. 50, pp. 387-410, Jul-Sep. 2006.

12. Wang and A. Chandrakasan. A $180 \mathrm{mv}$ fft processor using subthreshold circuit techniques. In Proc.IEEE ISSCC Dig. Tech. Papers, pages 229-293, 2004.

13. Tyler Thorp, Dean Liu and Pradeep Trivedi, "Analysis of blocking dynamic circuits", IEEE Transactions on VLSI Systems, pp. 744-749, 2003

14. Flautner, "Drowsy caches: Simple techniques for reducing leakage power", Proc. Int. Symp. Comput. Archit., pp. 148-157, 2002.

15. T. Cherpak, A. A. Barannik, Y. V. Prokopenko, Y. F. Filipov and S. A. Vitusevich, "Accurate microwave technique of surface resistance measurement of large-area HTS films using sapphire quasioptical resonator", IEEE Trans. Appl. Supercond., vol. 13, pp. 3570-3573, Jun. 2001

16. Vengatesan, k., kumar, a., naik, r., \& verma, d. (2018). Anomaly based novel intrusion detection system for network traffic reduction. In 2018 2nd international conference on i-smac (iot in social, mobile, analytics and cloud)(i-smac) i-smac (iot in social, mobile, analytics and cloud)(i-smac), 2018 2nd international conference on (pp. 688-690).

17. Department of electronics and communication engineering, sri satyasai university of technology and medical sciences, sehore, bhopal-indore road, madhya pradesh, india. Email address: satyant34@gmail.com

18. Department of electronics and communication engineering, sri satyasai university of technology and medical sciences, sehore, bhopal-indore road, madhya pradesh, india.

19. Mallikalava, v., yuvaraj, s., vengatesan, k., kumar, a., punjabi, s., \& samee, s. (2020). Theft vehicle detection using image processing integrated digital signature based ecu. In 2020 third international conference on smart systems and inventive technology (icssit) (pp. 913-918). 